Private production of public space. Private spaces for public use and planning incentives

\section{| RESUMEN |}

Existe un campo de estudio dedicado a espacios de uso público situados en terreno privado que han sido gestionados en el marco normativas de incentivos. ¿Qué motivación tienen los productores de estos espacios? ¡Es esencialmente posible que estos espacios contribuyan a la construcción de la vida pública de la ciudad?

\section{| ABSTRACT |}

There is a field of study dedicated to public use areas located on private land that have been managed under incentive regulation. What motivation do the producers of these spaces have? Is it essentially possible that these spaces contribute to the construction of public life in the city?

\title{
Producción privada de espacio público Espacios privados de uso público y la planificación por incentivos**
}

《Veredas porticadas», «galerías cubiertas» \y «patios» que articulan los interiores de manzanas forman parte de los espacios aparentemente públicos de la ciudad y tanto éstas como otras tipologías de espacios urbanos privados han sido ampliamente tematizadas en las últimas décadas (Sorkin, 1992; Low/Smith, 2006; Zukin, 2010; Siebel, 2003; Selle, 2003). El tema del uso público de espacios legalmente privados ha tomado hoy un lugar central en la discusión sobre espacio público, al punto que ha hecho necesario definir el límite que distingue el uso público del uso colectivo o privado y definir bajo qué circunstancias hay peligro de perder los derechos fundamentales democráticos en la esfera pública de la ciudad (Crawford, 1992; Sorkin, 1992; Soya, 1992).

En diversas ciudades del mundo, la génesis de muchos de los espacios privados de uso público está asociada al Incentive Zoning, norma urbana de incentivo aplicada a proyectos inmobiliarios, que consiste en la bonificación de una mayor constructibilidad a cambio de ceder al uso público una cantidad de superficie en el lote privado. En ciudades como Boston, Nueva York, Hartford, San Francisco, Seattle, Tokio y Yokohama se ha aplicado el Incentive Zoning para generar espacios de uso público (Svirsky, 1970; Lassar, 1982; Getzels/Jaffe, 1988; Babcock/Larsen, 1990; Weiss, 1992; LoukatouSideris/Baneriee, 1992; Cullingworth, 1997; Dimmer, 2006; Fujii/Okata/Sorensen, 2007).

Nueva York es, sin embargo, la ciudad donde fue concebido originalmente este mecanismo en 1961 (Weiss, 1992; Weaver/Babcock, 1979). La incorporación de este nuevo concepto de canje está intrínsecamente relacionada con la historia de la Ordenanza de Zonificación de

* Arquitecta de la Pontificia Universidad Católica de Chile, PUC, y Doctora en Arquitectura (Dr., Ing.) por la Universidad Tecnológica de Berlín, Alemania. Es académica e investigadora de la Universidad Andrés Bello, UNAB, y académica de la PUC. Sus investigaciones en la UNAB y FONDECYT se centran en el análisis de la legislación urbana como portadora de imagen ciudad. Se ha centrado en el tema del estatus jurídico del espacio público, particularmente, la figura legal de aquellos espacios de uso público y colectivo que se encuentran en suelo privado, así como en los instrumentos participativos que promueven el desarrollo sustentable de los barrios. Ha escrito diversos artículos y presentado ponencias en Chile y el extranjero sobre el espacio público y legislación urbana Es coautora del libro Santiago Subterra, publicado por Ediciones FAAD UNAB-COOP (2009). Actualmente es miembro de la red de investigadores StaRS international - The Development of Privately Owned Public Space. A comprehensive, co-operative study in North and Latin America, Asia, Australia, Africa, and Europe.

** Paper titulado «Publicly Used Commercial Space and Incentive Zoning: Opportunities and Constraints Arising from the Private Production of Public Spaces in Santiago, Chile» presentado en el $5^{\text {th }}$ International Conference «Planning Law \& Property Rights». University of Alberta, Canadá en mayo de 2011 . Corresponde a los hallazgos de la investigación «Producción privada de espacios de uso público. Los casos resultantes de la normativa de incentivo de Providencia entre los años 1976 y 2006», investigación financiada por CONICYT - Fondecyt Iniciación Nº 11090407, cuya investigadora responsable es Elke Schlack. 
1. Mayor constructibilidad a cambio de cesión de espacio al uso público. Normativa de incentivo a la creación de pasajes por el interior de la manzana, Art. $48 d$ del Plan Regulador de la Comuna de Providencia (Fuente: Schlack, E., 2011; dibujo: C. Ivovich y M. Urzúa).

2. Art. 460 Ordenanza General de Construcciones y Urbanización 1936 (Fuente: Schlack, E., 2010; dibujo esquema: M.N. Hinojosa)

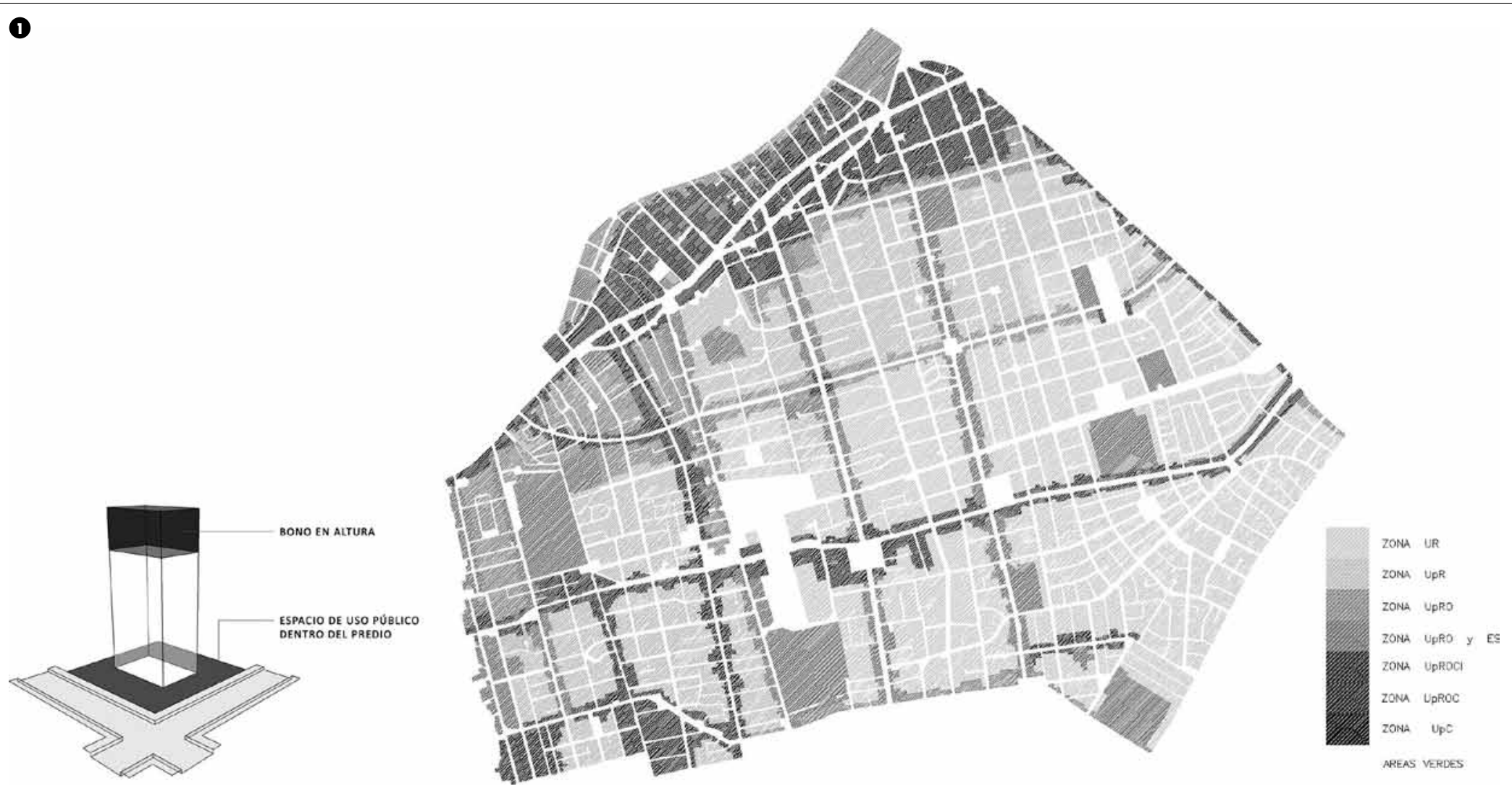

Nueva York de 1916 que en 1960 sufre un importante cambio: en vez de regular la altura de los edificios mediante un «máximo volumen envolvente» definido por planos imaginarios inclinados (set back) (Kayden, 2000; Pegels, 2004), se permite construir edificios de mayor altura sin set backs que, a cambio, ceden un espacio libre en el terreno tal como lo propone el edificio «Seagram» del arquitecto Mies van der Rohe en 1957 (Pegels, 2004). Desde 1961 por cada metro cuadrado cedido como espacio de uso público, se bonificaban diez metros cuadrados de constructibilidad adicional a los edificios ubicados en la zona del plan (Pegels, 2004). Según lo que dictaba esta normativa, el gestor inmobiliario asumía la construcción y mantención de este espacio, comprometiéndose a dejarlo accesible al público (Pegels, 2004; Kayden, 2000). En la medida que evolucionó la ordenanza de 1961, las tipologías iniciales (la plaza y la arcade) fueron complementadas con nuevas tipologías: side walk widening, elevated plazas, sunken plazas, though block galleries, etc.) (Whyte, 1980; Kayden, 2000; Pegels, 2004).
La evaluación de esta política de incentivos muestra que de 1961 a 2000 se crearon 503 Privately Owned Public Spaces o POPS, lo cual equivale a un diez por ciento del Central Park (Kayden, 2000). Si bien los resultados cuantitativos parecen alentadores, los resultados cualitativos hacen dudar de este mecanismo, según un grupo liderado por Whyte y Kayden. La evaluación de la calidad espacial y del uso de estos 503 espacios se realizó sobre la base de asignarles las siguientes categorías de calidad: espacios buscados explícitamente como destino (destination space), espacios atractivos para la vecindad (neighborhood space), espacios de breve detención (hiatos space), espacios de paso (circulation space) y espacios inutilizados (marginal space). El resultado de la evaluación fue el siguiente: sólo 15 espacios fueron evaluados como «destination places» (3\%), 66 como «neighborhood spaces» (13\%), 104 como «hiatus spaces» (21\%), 91 como «circulation spaces» (18\%) y 207 como «marginal spaces» (41\%) (Kayden, 2000).

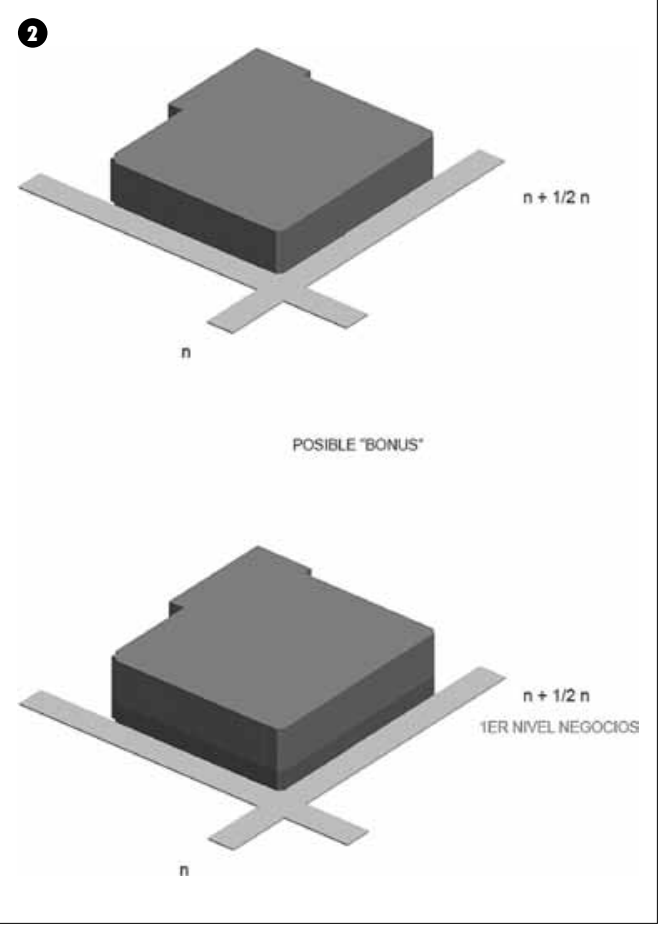



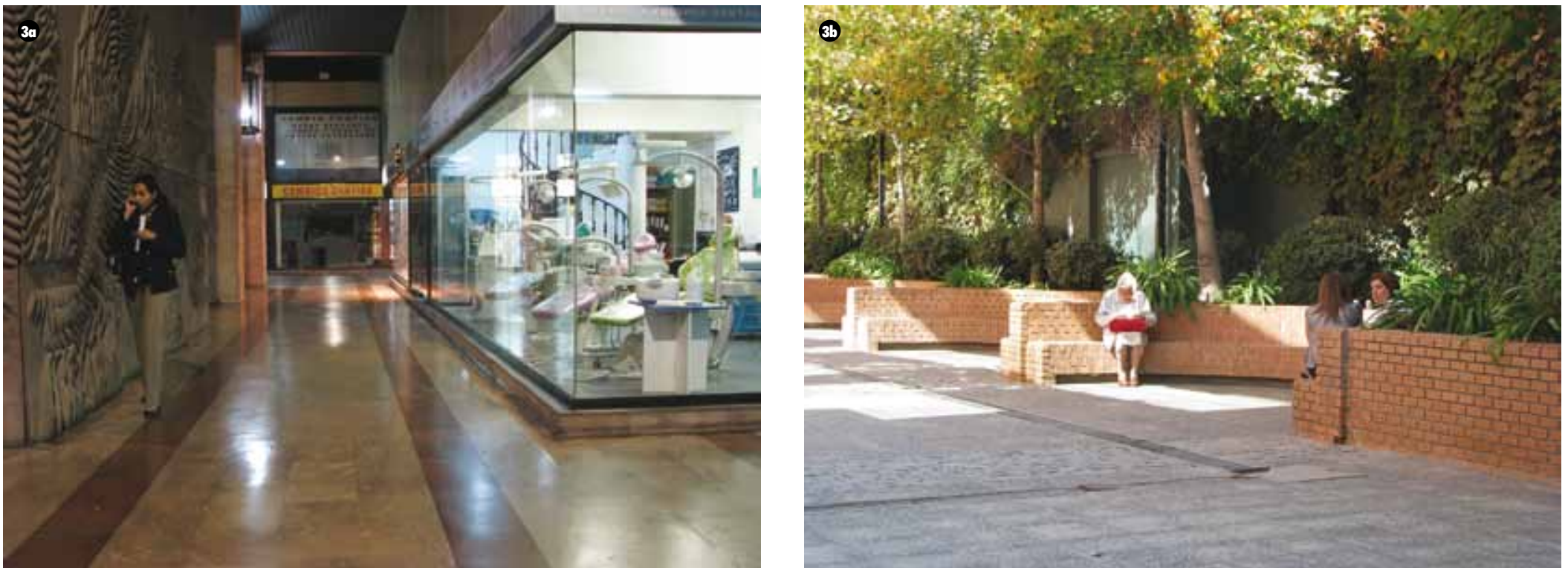

Diversos autores que estudian el caso de Nueva York han buscado entender los motivos de los negativos resultados. Por un lado, se ha analizado el fenómeno de los POPS asumiendo que el mal resultado es efecto colateral de la maximización de la rentabilidad inmobiliaria que negligentemente descuida la creación de espacios atractivos al uso público (Whyte, 1988). Por otro lado, se sostiene la tesis de que tras espacios desolados podría estar la acción intencional del gestor inmobiliario, que se esfuerza por producir espacios que repelen el uso público y más bien buscan un espacio con cualidades escultóricas y paisajísticas (Smithsimon, 2008).

No sólo en América del Norte y en Asia, sino que también en Sudamérica, particularmente en Santiago de Chile, se tiene experiencia con el uso del Incentive Zoning. La particularidad de este caso, operativo desde 1972, es que se plantea en el contexto de la renovación urbana, como un instrumento para guiar la transformación de un sector predominantemente residencial en un subcentro comercial. Esta política urbana es exclusiva de la comuna de Providencia y tiene el objetivo principal de planificar simultáneamente la volumetría de los edificios y el espacio libre entre éstos. El espacio libre se concibe como una red peatonal articuladora del espacio urbano de este importante subcentro de la ciudad.
La falta de reservas de suelo y de financiamiento para implementar espacios peatonales llevó tempranamente a los planificadores de Providencia a considerar la posibilidad de generar superficies fuera del ámbito público (Bannen, entrevistas 2001-2003; Mora/Zapata, 2004; Schlack, 2008). Si bien el mecanismo del Incentive Zoning fue aplicado por primera vez en Chile a través de la normativa comunal de Providencia en 1972, en Santiago ya existía el antecedente del incentivo a la construcción de galerías comerciales en los dos primeros pisos de las manzanas compactas del centro desde la década de los treinta (ver Art. 460 Ley y Ordenanza General de Construcciones y Urbanizaciones de 1936). Esta norma contribuyó a la consolidación de una extensa red de galerías y portales peatonales a la manera de las galerías parisinas descritas por Walter Benjamin.

\section{EL ESPÍRITU DEL PLAN DE PASAJES DE PROVIDENCIA}

El plan que ordena la planificación por incentivos en Providencia tenía objetivos urbanísticos muy definidos: promover «un Centro Urbano en densidad...» en que hubieran espacios "que posibilitaran el encuentro», donde los peatones pudiesen recorrer espacios junto a la calle y por el interior de las manzanas. El comercio se concibe como el uso que construye los bordes de un espacio «intransablemente» público (Bannen, 2008). Los casos que coinciden mejor con el espíritu de este plan tienen las cualidades de un comercio que complementa el espacio público y no de artefactos comerciales segregados de los espacios de la ciudad. La idea del plan es que los espacios estuvieran fuertemente vinculados a las infraestructuras de transporte y redes peatonales y que configurara espacios cotidianos de una gran vitalidad (Bannen, 2008).

El plan ha tenido un fuerte impacto en las cualidades del espacio urbano de la comuna. Se ha comprobado la existencia de 62 edificios que han aportado con espacios públicos acogiéndose a la norma de incentivos. Gran parte de ellos se ubica en la zona definida como «predominantemente comercial» en el Plan Regulador de la comuna, la zona con mayor intensidad de suelo. Sumados, estos espacios cubren una superficie total de aprox. $60.000 \mathrm{~m}^{2}$, lo que constituye el $3 \%$ de las áreas verdes de la comuna (Schlack, 2010).

\section{CONTEXTO Y FASES DEL PLAN}

Cuando se establecen los estudios preliminares para dicho plan (entre 1967 y 1972),

Providencia estaba viviendo una fuerte 
4a. Limitada continuidad con el espacio público circundante. El edificio caracol «Dos Providencias» de 1977 y sus rampas de circulación vertical (Fuente: Schlack, E., 2010). 4b. Continuidad con el espacio público. El pasaje «Santa Magdalena» entre los edificios «Las terrazas de Providencia» y «Dos Providencias» ambos de de 1977 (Fuente: Schlack, E., 2010).
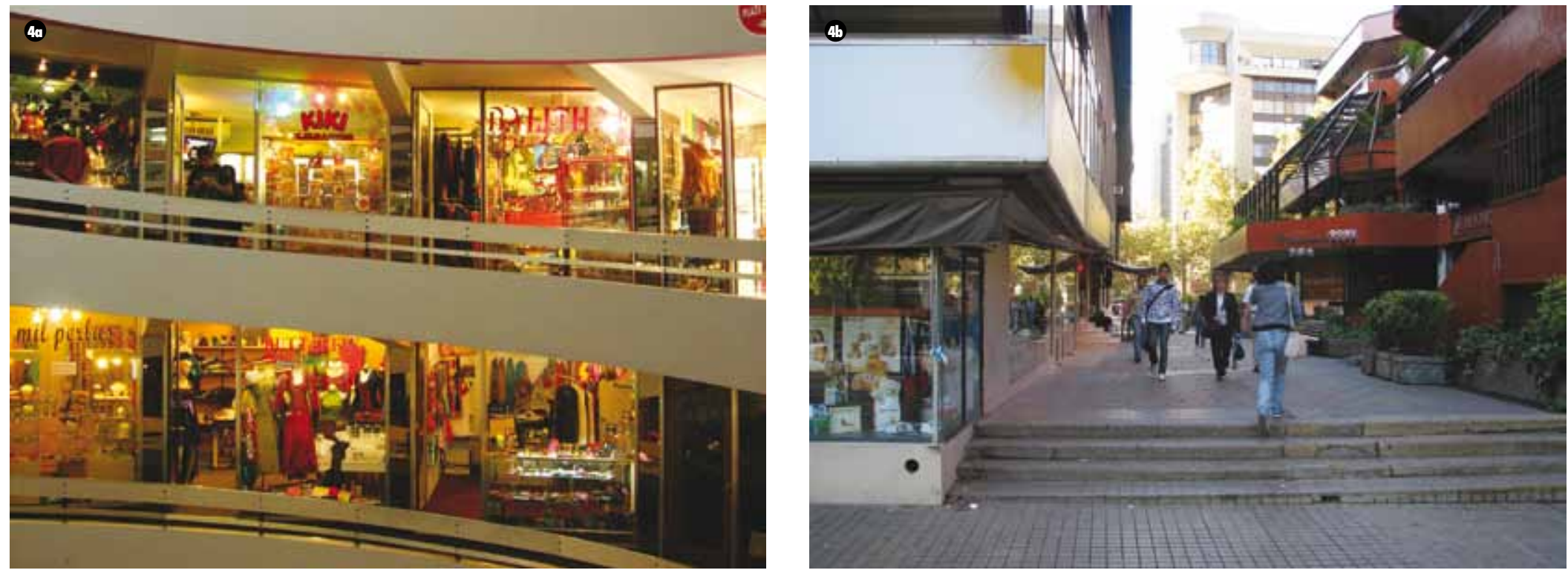

transformación que la convertía en el nuevo subcentro comercial de Santiago, desplazando el antiguo centro comercial y administrativo ubicado en el centro histórico de la ciudad. Ya en 1967 el sector presentaba una importante renovación consistente en la demolición de antiguas casonas, la fusión de predios de 500 $\mathrm{m}^{2}$ a predios de $2.000 \mathrm{~m}^{2}$ y la construcción de edificios entre 5 y 8 pisos. En 1972 se aprueba el plan comunal para la «Nueva Providencia», que contempló el establecimiento de una red de pasajes por el interior de las manzanas en renovación y la apertura de nuevas vías vehiculares estructurales para la ciudad entera.

En una primera fase, entre 1972 y 1989, los instrumentos para implementar el plan fueron los llamados «planos seccionales», los que definían la ubicación de los pasajes y patios interiores de las manzanas. El premio por aportar espacios al uso público consistía en una mayor constructibilidad (entre el $25 \%$ y el $50 \%$ adicional a la norma vigente) y la posibilidad de alterar la tipología edificatoria prevista para dicha zona (Norma de Conjunto Armónico) (Schlack/Vicuña, 201 la). La forma en que los pasajes se diseñaban finalmente, se consensuaba informalmente caso a caso entre el planificador y el proyectista, instancia que hacía posible transmitir el espíritu del plan a cada uno de los arquitectos de los edificios que se construyeron bajo esa modalidad. El aumento de constructibilidad en los casos de esta primera fase nunca superaba un Coeficiente de Constructibilidad de 7

En una segunda fase, de 1990 en adelante y tras una serie de cambios de orden orgánico a nivel nacional y comunal, el plan se implementa a través de una norma escrita que establece el aumento de coeficiente de constructibilidad en función de los metros cuadrados cedidos al uso público. De esta forma, por cada $\mathrm{m}^{2}$ cedido al uso público el edificio podía ganar $5 \mathrm{~m}^{2}$ adicionales en constructibilidad. La definición de aspectos cualitativos ya no se realiza caso a caso, sino que se reduce a una condición: los pasajes debían conectar calles o pasajes existentes o propuestos sin establecer disposiciones de orden espacial (Artículo 48d, hoy artículo 3.3.06 de la Ordenanza del Plan Regulador de Providencia).

Si bien en dicho artículo se establece que la condición para otorgar más derechos de construcción es que estos espacios al nivel de la calle deben permitir el libre paso peatonal, no se establece la forma en que esta condición de uso público adquiere su condición de legalmente vinculante. La inscripción legal no es parte del requisito para otorgar el premio en la norma de incentivo y muchas veces el «tránsito público» de un espacio queda sólo inscrito en el permiso de edificación, lo cual no tiene ninguna validez en el momento de querer controlar o penalizar el incumplimiento de esta condición. El aumento de constructibilidad llegaba, gracias a esta modalidad, al $200 \%$ de la constructibilidad permitida, llegando a valores de Coeficiente de Constructibilidad $=9$.

Debido a limitaciones que establece la normativa chilena referente a las disposiciones de diseño que puede establecer un Plan Regulador comunal, hasta ahora no se han incorporado nuevas normativas en el sentido de regular con más precisión la forma de los espacios ni las tipologías o programas asociados, como en Nueva York. La normativa chilena no concibe la posibilidad de regular el espacio libre más allá de las normas de altura de edificación, Coeficiente de Constructibilidad, ocupación de suelo, forma de agrupación, distanciamientos y rasante, que en el fondo son normas que definen la forma y ubicación de lo construido en el terreno y sólo indirectamente la forma del espacio libre (Méndez, 2010).

\section{EL COMERCIO QUE SURGE DE LA NORMA DE INCENTIVOS EN PROVIDENCIA}

Los espacios urbanos surgidos a partir de la norma de incentivos en Providencia presentan sustanciales diferencias respecto de los casos en la ciudad de Nueva York. En Providencia, 
5a. Distinguirse de la materialidad y mobiliario de la calle. Conjunto «Drugstore» originalmente de 1967, remodelado en 2006 con una lógica distinta a la calle (Fuente: Schlack, E., 2010). 5b. Similitud con la materialidad y mobiliario de la calle. Pasaje junto el edificio «Carlo Magno» de 1977 (Fuente: Schlack, E., 2010).
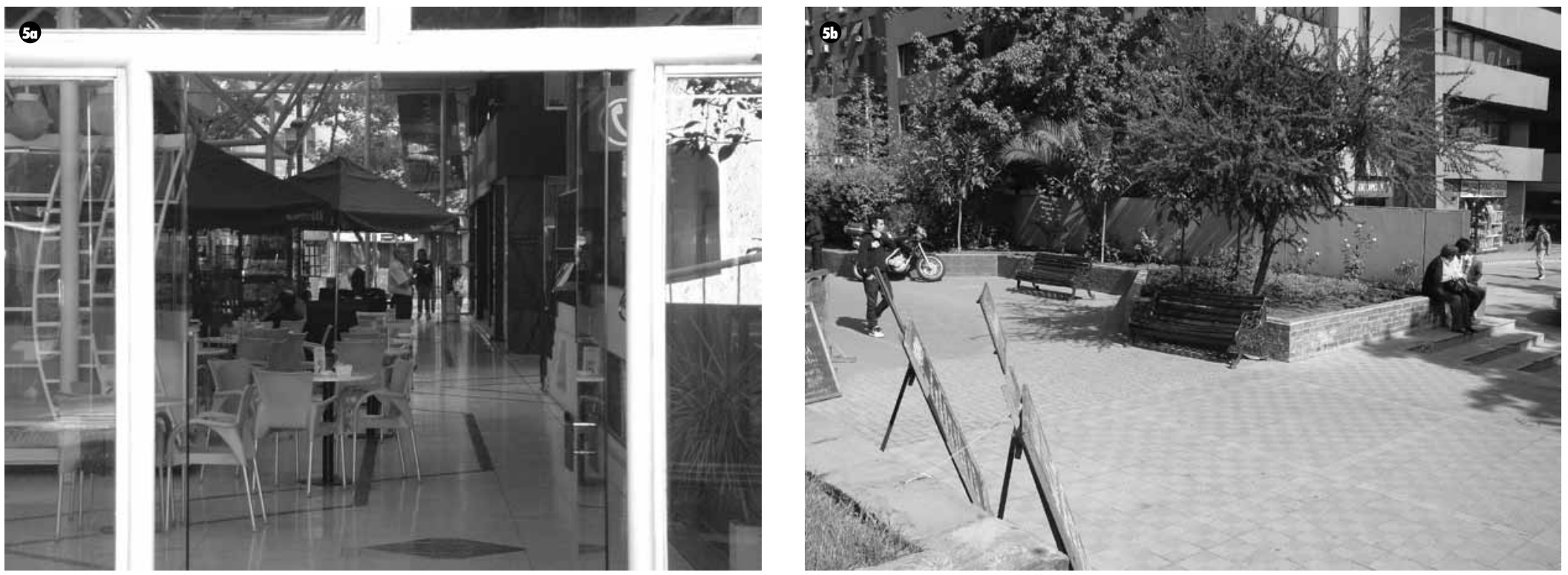

la mayoría la constituyen espacios privados rodeados por comercio, ya sea como locales a nivel calle o incluso en muchos casos como edificios completos destinados al comercio, como la tipología del «Caracol» o el «Centro Comercial» con galerías. Es por esto que no cabe duda que en Providencia, al contrario de los espacios desolados de Nueva York, encontramos en gran medida espacios de gran afluencia peatonal.

\section{A diferencia de la pregunta que hace} Smithsimon sobre los casos de Nueva York, en relación a los casos de Providencia es pertinente preguntar por el interés que tiene el gestor inmobiliario por producir espacios de uso público en las inmediaciones de su proyecto. Partimos de la hipótesis de que a los desarrolladores les parece atractivo construir espacios de uso público más allá del premio que reciben en forma de constructibilidad adicional, porque éstos le otorgan una gran afluencia de público a los espacios comerciales ubicados en el nivel calle de sus edificios. Más aun, la forma y calidad de estos espacios de uso público estará condicionada por intereses espaciales y programáticos muy determinados: promover el acceso al comercio a través de tipologías propias de espacios comerciales y de un diseño del espacio y el equipamiento (iluminación, asientos, materiales) que estará determinado por una estética que el gestor considera apropiada para promover el comercio.

\section{DESCRIPCIÓN DE LOS HALLAZGOS DE LA INVESTIGACIÓN}

\section{CUALIDADES FÍSICO-ESPACIALES Y LA POSIBILIDAD DEL USO PÚBLICO}

De los 62 casos resultantes del Plan de Providencia, 30 contienen comercio en su programa y 15 consisten en programas predominantemente comerciales asociados a tipologías como «Centros comerciales», «Caracoles», "Galerías», etc. (Schlack, 2011 b). La vocación comercial del centro de la comuna de Providencia se mantiene en crecimiento entre los años setenta y fines de los ochenta. Al menos 15 edificios de comercio son construidos paralelamente al plan de pasajes en esas dos décadas. Una inflexión en el desarrollo comercial de Providencia la constituyen la aparición del primer Centro Comercial de Santiago, el «Apumanque», en un nuevo subcentro comercial ubicado en Las Condes (en 1980) y la construcción del primer «Mall» de Chile (el «Parque Arauco»), en 1981, también en la comuna de Las Condes. Después de la aparición de estos centros, en Providencia sólo se registra la construcción de tres edificios nuevos, así como la reconversión de dos edificios antiguos dedicados al comercio y acogidos a la norma de incentivos.

Las entrevistas realizadas a los gestores y sus arquitectos que construyeron espacios comerciales mediante el mecanismo de incentivos permiten visualizar que la mayoría de ellos estima ventajoso ceder espacio al uso público, ya que lo relacionan con la mayor afluencia de público a su comercio, tal como se planteó en la hipótesis. La manera en que quisieron caracterizar los espacios en términos de su expresión físico-espacial y de su uso se diferencia caso a caso. Sin embargo, en las entrevistas se destacan aspectos vinculados al diseño de los espacios que dicen relación con la cualidad de paso (y no permanencia) de éstos, la continuidad peatonal entre edificio y ciudad, la diferenciación físico-espacial respecto de la calle y el manejo del control de ellos. Estos aspectos son particularmente importantes para determinar si promueven o repelen el uso público.

En primer lugar está el tema del paso versus la permanencia. En los casos analizados podemos encontrar espacios que sólo permiten el paso y tienen forma de pasillos flanqueados por vitrinas en ambos lados (ej. edificio Forum). Otros casos, en cambio, disponen de algunos espacios para la permanencia, lo que se expresa a través del tamaño mayor del espacio, de cualidades agradables para la permanencia y de mobiliario como bancas, etc. (ej. Plaza Lyon y Paseo Las Palmas). La fuerte vocación de los espacios comerciales para constituir espacios de tránsito frente a las vitrinas es, sin duda, una limitación al uso público, uso inherente a su condición 
6a. Explícito control privado. Conjunto «Patio Bellavista» exhibe sus guardias con uniformes distintivos (Fuente: Schlack, E., 2010) 6b. Formas de control social (público) ejercida por paseantes, habitantes, vecinos, comerciantes ambulantes, mendigos, etc. Edificio "Costanera 2» de 1978 (Fuente: Schlack, E., 2010).
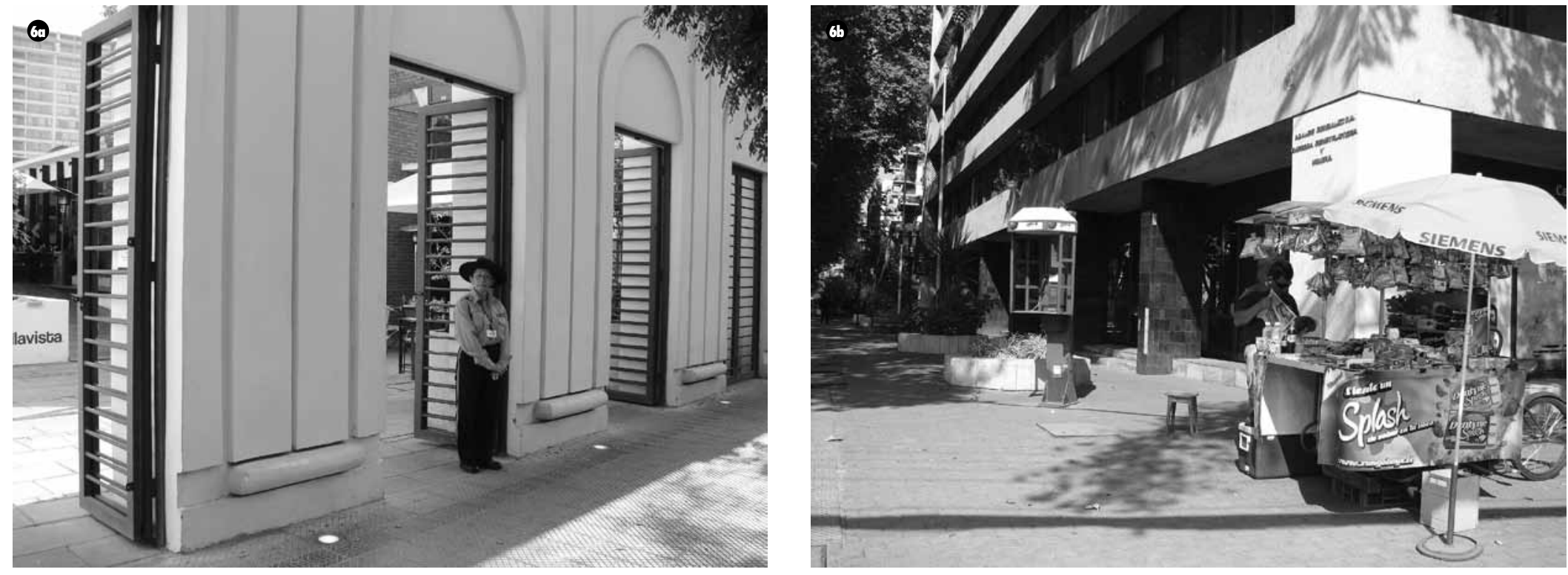

de estar y permanecer, permitiendo encuentros inesperados y espontáneos (comparar con indagaciones de Wehrheim, 2009; Gehl, 2006).

En segundo lugar está el aspecto de cómo está concebida la conectividad con el espacio público circundante de edificios dedicados al comercio. En los casos analizados se distinguen aquéllos que se constituyen como edificios específicos para el consumo, cerrados en sí mismos (ej. Multitienda Falabella, Dos Caracoles). Sin embargo, hay otros espacios que se conectan y articulan con la infraestructura urbana (ej. Plaza Lyon, Paseo Las Palmas). Los edificios cerrados en sí mismos están presentes en todas las décadas y constituyen tipologías diversas: desde el "Caracol» hasta la "Multitienda», sin embargo, los edificios conectados a la ciudad son una expresión propia de la década de los setenta y ochenta, de los cuales podemos mencionar diversos espacios como el «Interprovidencias», el "Centro Nuevo» y el «Dos Providencias», los que ayudan a conformar espacios junto con zonas peatonales, mezaninas de estaciones de Metro, etc. El sentido original del plan de Providencia era juntar el comercio con la vida pública cotidiana, su vinculación con las infraestructuras. La construcción de redes y buenas continuidades apoya la posibilidad de un uso público, ya que hace accesible el espacio a un número mayor de personas.
En tercer lugar se destaca el tema de cómo se diferencia o se asemeja el espacio privado al espacio de la calle. En esto se distinguen muchísimo el grueso de los casos de los setenta y ochenta de los recientes. Es posible notar que los espacios de las primeras décadas usan el recurso de ser «similares a la calle» en términos de sus materiales y mobiliario (ej. plaza del Sol, Fallabella exterior). Los casos recientes usan con intención el recurso de diferenciarse de la calle, elaborando mediante materiales y mobiliario cualidades propias del «interiorismo» y generando espacios diferenciados, lo cual no sólo es simbólico, sino que real porque muchas veces va de la mano con situar en estos espacios gastronomía y tiendas exclusivas (ej. «Galería Drugstore», Torre París). El recurso simbólico de que un lugar aparezca diferenciado de la calle hace que el espacio atraiga menos el uso público y promueva más el uso de determinados grupos que se sienten cómodos con el requisito de la exclusividad.

Por último, destaca el aspecto relacionado con la forma en que se conciben los espacios y las medidas de control para manejar la seguridad en ellos. La sensación de inseguridad ha crecido en Chile desde los años setenta. Eso se puede seguir en la forma que los espacios más antiguos manejan su apertura y conexión con los espacios públicos del entorno, veredas, etc. Se trata de espacios en los que no se perciben sus puertas nocturnas y que establecen una continuidad visual con las veredas (ej. Pasaje Divina Providencia, Interprovidencias, Dos Providencias). A diferencia de esto, los espacios más recientes evidencian su situación "controlada» a través de cerramientos de vidrio, barreras magnéticas a traspasar, etc. (ej. Drugstore, Fallabella). Si bien en ambos el control social puede existir a través de la presencia de los locatarios o por los restaurantes que sacan sus mesas al espacio abierto, el control privado mediante guardias o cámaras de vigilancia es parte de la estética explícita del espacio. El comercio tiene la gran virtud de que puede, bien manejado, resolver el tema de la seguridad mediante el control social. Esto depende, eso sí, de la modalidad en que comercio y espacio se relacionan. Si se integran, la seguridad del espacio se ve favorecida por la actividad comercial, si el espacio se segrega, es necesario recurrir a modos de vigilancia y control para mantenerlo seguro.

\section{COMENTARIO A LA DEFINICIÓN DE LAS CUALIDADES ESPACIALES PÚBLICAS DE LOS ESPACIOS}

Si bien es cierto que la normativa de incentivos de Santiago aun podría ser desarrollado en el sentido del caso de Nueva York, donde se han estipulado tipologías diferenciadas para situaciones urbanas distintas e incluso se podría avanzar en la formulación de normas 
urbanísticas que precisen la regulación del espacio libre entre lo construido, podemos constatar que las prescripciones formales no son la única clave para regular este tipo de espacios. Sabemos a través de la investigación realizada que los gestores y sus arquitectos pueden priorizar ciertas expresiones físico-espaciales en la construcción de espacios de uso comercial que pueden disminuir el «uso público» de un lugar.

\section{COMENTARIO A LA DEFINICIÓN DEL ESTATUS LEGAL DEL USO PÚBLICO DEL ESPACIO}

La actual regulación de espacios privados de uso público en Chile no ha resuelto aún su estatus legal de uso público. Sólo algunos casos cuentan con una "servidumbre de tránsito» inscrita en el Conservador de Bienes Raíces, lo cual permite la existencia jurídica del paso de un predio a otro y la permanencia en el tiempo del «gravamen» de paso público. La mayoría de los casos, sin embargo, no garantizan el paso a lo largo del tiempo y de todo el espacio cedido (ej. Espacio de Multitienda Falabella, espacio del Patio del Sol en que se ubican mesas de restoranes).

La inscripción legal como «servidumbre de paso público» podría ser parte del requisito para otorgar el premio en la norma de incentivos, tal como sucede al menos en la legislación alemana, en que es posible gravar el uso público en un Plan Seccional como es el caso de la zona de la galería «Potsdamer Arkaden» en el desarrollo llamado Potsdamer Platz (Schlack, 2009). Junto con definir el uso público, es necesario definir quién tiene tuición sobre la mantención y control del espacio. En la visión de los gestores de los casos analizados éste es un tema en el que es necesario revisar, hasta qué punto debiesen hacerse cargo privados y municipio complementariamente.

\section{CONCLUSIÓN}

Sin lugar a dudas, la aplicación de mecanismos de incentivo para generar espacios de uso público aparece, ante los disminuidos fondos municipales, como una atractiva forma de producir espacios públicos; sin embargo, también es importante tener en cuenta las limitaciones que tiene esta forma de producción privada de espacios. La planificación por incentivos es una fórmula que debe ser necesariamente complementada por otros mecanismos que permitan que la ciudad se conforme como un mosaico de diversas formas de espacio público: espacios de representación política, plazas residenciales, subcentros comerciales, entre otros. Esta necesidad de diferentes frentes para gestionar el espacio público en la ciudad está presente en el planeamiento urbano de la comuna de Providencia: la gestión de espacios privados de uso público va de la mano con la gestión de parques integrados y la implementación de edificios públicos que catalizan el uso público de parques comunales.

La construcción de espacios de uso público por privados no es sólo de incumbencia privada. El municipio es el que finalmente se hace cargo a largo plazo de los efectos de decisiones privadas en la esfera pública. Por un lado, tenemos los "Caracoles», producto inmobiliario recurrente durante la crisis económica vivida en Chile en los años '70 y '80, que hoy se presenta como un tipo de edificio difícil de renovar y readecuar, dada su estructura de tenencia y su configuración espacial. Por otro lado, se gestaron en la década de los años '90 una serie edificios cuyos primeros pisos si bien contienen programa de comercio, están situados donde la demanda comercial no es suficientemente intensa. El resultado son primeros pisos deshabitados que contribuyen a desarrollar espacios poco transitados, sin vitalidad y desprovistos de control social.

Por último, la investigación devela la desconfianza que algunos arquitectos y gestores declaran en relación a hacerse cargo del espacio público. No es tan claro, cuál es el manejo apropiado del diseño del espacio para promover el uso público sin recurrir a fórmulas de la «interiorización» y el control privado. Anticipar y guiar el desarrollo de los espacios públicos por parte de los municipios es tarea compleja considerando que los gestores involucrados consideran que es un territorio incierto o que recurren a la «privatización» como receta para sortear el problema. Para promover la creación de espacios de uso público, independiente de quién los produzca y quién los mantenga, es necesario seguir discutiendo la forma de compatibilizar los intereses individuales con el bien público: el uso público de los espacios.

\section{BIBLIOGRAFÍA}

Babcock, RF; Larsen, WU. Special Districts: The Ultimate in Neighbourhood Zoning. Cambridge, MA.: Lincoln Institute of Land Policy, 1990

Bannen, G. «El comercio en Providencia». En: Revista CA N ${ }^{\circ} 72$, Edición CA, Santiago, 1993 (también: CA N²5, 1979; CA N 27, 1980; CA N 57, 1989; CA N ${ }^{\circ} 58$, 1989).

Bannen, P; Chateau, F. «La ciudad de Providencia en la obra de Germán Bannen». Serie Monografías de Arquitectura Vol. 3. Santiago de Chile: ARQ, 2007.

Berding, U; Havemann, A; Pegels, J; Perenthaler B. Stadträume in Spannungsfeldern. Plätze, Parks und Promenaden im Schnittbereich öffentlicher und privater Aktivitäten. Dorothea Rohn.

Crawford, M. «The World in a Shopping Mall». En: Sorkin, M. Variations on a theme park: The new american city and the end of public space. New York: Hill and Wang, 1992.

Dimmer, Ch. Renegotiating Public Space. A Historical Critique of Modern Public Space in Metropolitan Japan and its Contemporary Re-valuation. Tokyo: University of Tokyo, 2006.

Dimmer, Ch. «Privately owned Public Space: Japan's uncontested corporate Commons». En: Schlack, E. Espacios privados de uso público. Santiago de Chile: UNAB-Coop. 2012.

Fujii, S; Okata, J; Sorensen, A. «Inner-city redevelopment in Tokyo. Conflicts over urban places, planning governance and neighbourhoods». En: Sorensen, A.; Funck, C. Living cities in Japan: citizens' movements, machizukuri and local environments. London: Routledge, 2007.

Getzels, J; Jaffe M. «Zoning bonuses in central cities». PAS Report N ${ }^{\circ} 410,3-4$. Chicago: American Planning Association, 1988.

Habermas, J. Conciencia moral y acción comunicativa. Barcelona, Península, 1991.

Havemann, A; Selle, K. Plätze, Parks und Co. Stadträume im Wandel - Analysen, Positionen und Konzepte. Dorothea Rohn, 2010. 
Lassar, T. Carrots and sticks: New zoning downtown. Washington, DC: Urban Land Institute, 1982.

Loukaitou-Sideris, A; Baneriee, T. Private production of downtown public open space: Experiences of Los Angeles and San Francisco. Los Angeles: School of Urban and Regional Planning, University of Southern California, 1992.

Kayden, J. Privately Owned Public Space: The New York city experience. New York: John Wiley, 2000.

Low, S; Smith, N. The politics of public space. New York: Routledge, 2006.

Méndez, M. «Entrevista realizada por la autora» (2010), se publicará en: Schlack, E. Espacios privados de uso público. Santiago de Chile: UNAB-Coop., 2012

Mitchell, D. The right to the city: social justice and the fight for public space. New York: Guilford Press, 2003

Mitchell, D; Staeheli, L. «Clean and safe? Property redevelopment, public space and homelessness in downtown San Diego». En: Low, S.; Smith, N. The politics of public space. New York: Routledge, 2006.

Mora, R; Zapata, I. «El comercio, espacio público y gestión local: el caso de Providencia en Santiago de Chile». Revista Urbanismo $N^{\circ}$ 9, 2004

Pegels, J. «Privately Owned Public Space New York Citys. Erfahrungen im Umgang mit öffentlich nutzbaren Räumen, die sich in privatem Besitz befinden. |». Architektur und Planung $N^{\circ}$ 1, 2004, Dissertationen an der Fakultät für Architektur der RWTH Aachen.

Pegels, J. «Privately Owned Public Space como desafío. La cambiante historia del incentive zoning en la ciudad de Nueva York». En: Schlack, E. Espacios privados de uso público. Santiago de Chile: UNAB-Coop., 2012.

Plan Regulador de Providencia, Decreto Supremo $N^{\circ} 424$ del Ministerio de Vivienda y Urbanismo del 24-1-1976.

Rosas, J. Manzana y tipo edificatorio en transformación. Barcelona: Escuela Técnica Superior de Arquitectura de Barcelona, 1986.

Schlack, E. «Städtebaurecht und öffentlicher Raum». Tesis doctoral presentada en la Universidad Tecnológica de Berlín, 2009.

Schlack, E. Documento final. La gestión de espacios de uso público en la planificación comunal: El Plan de espacios de uso público en la comuna de Providencia. Investigación financiada por UNAB N ${ }^{\circ}$ DI 44-09/JM 2010.

Schlack, E; Vicuña, M. «Highly influential normative components regarding the new morphology of Metropolitan Santiago: a critical review of 'Harmonic Set'». Revista EURE N ${ }^{\circ} 111$, Vol. 37, mayo 2011, 131 166 (2011a).

Schlack, E. Documento final. Producción privada de espacios de uso público. Los casos resultantes de la normativa de incentivo de Providencia entre los años 1976 y 2006. Investigación financiada por CONICYTFondecył Iniciación N ${ }^{\circ} 11090407$ investigación 2009-2011 (2011b).

Schlack, E. Espacios de uso público. Santiago de Chile: UNAB-Coop., 2012 (2011c).

Selle, K. «Was ist los mit den öffentlichen Räumen». Informes AGB № 49 2, 2003. Aachen, Dortmund, Hannover. Dortmunder Vertrieb für Planungsliteratur.
Siebel, W. «Vom Wandel des öffentlichen Raumes». En: Wehrheim, J. Shopping Malls. Interdisziplinäre Betrachtungen eines neven Raumtyps. Wiesbaden: VS Verlag, 2007.

Smithsimon, G. «Dispersing the crowd: bonus plazas and the creation of public space». Urban Affairs Review 2008; 43:325

Smithsimon, G. «The Imperial City as Seen from the Plaza: New York's Regimes of Private Control». En: Schlack, E. Espacios de uso público. Santiago de Chile: UNAB-Coop., 2012

Sorkin, M. Variations on a theme park: The new american city and the end of public space. New York: Hill and Wang, 1992.

Svirsky, PS. «San Francisco: The downtown development bonus system». En: The new zoning: Legal, administrative, and economic concepts and techniques. 1970; 139-58.

Weaver, C; Babcock, R. City Zoning. Chicago: Planners Press, 1979.

Weber, M. Economía y sociedad, Tomo II - La dominación no legítima (tipología de las ciudades). México: Fondo de Cultura Económica, 1964 (1922 texto original).

Wehrheim, J. Shopping Malls. Interdisziplinäre betrachtungen eines neuen Raumtyps. Wiesbaden: VS Verlag, 2007.

Weiss, M. «Skyskraper Zoning. New Yorks's pioneering role». APA Journal 201, 1992.

Whyte, WH. The social life of small urban spaces. Washington, DC: The Conservation Foundation, 1980.

Zukin, S. Naked City. The Death and Life of Authentic Urban Places. Oxford: University Press, 2010. 